of bone lying on the outer side of it. The wound must now be tamponed, and not stitched.

Our author also suggests that a flap be made from the cutaneous lining of the auditory canal, corresponding with that part of the posterior wall which has been chiselled away, and pushed into the antrum mastoideum, so as to introduce into this cavity healthy epidermis, and thus secure a more rapid sheathing of this large osseous space, and institute a permanent fistulous opening between antrum and auditory canal, through which the condition of the antrum can always be inspected.

\title{
Experimental Avulsion of the Stapes in Animals.
}

DR. RICHARD BoTEY, of Barcelona, read a paper on this subject at the International Congress of Berlin. He was induced to make his experiments by an accident which happened while he was operating upon a patient, a man, thirty years old. It appears that while endeavoring to perform mobilization of the stapes for relief of deafness and tinnitus aurium, he pulled out the major part of the stapes, and the peri-lymph escaped from the vestibule and labyrinth. The ear was at once mopped out with an antiseptic, and the patient sent home with orders to remain there until Dr. Botey called the next day. Upon visiting the patient the next day the hearing was found to be improved, and the tinnitus had entirely ceased.

Dr. Botey then began experiments upon animals to discover (1) whether animals can hear without a stapes; (2) whether evulsion of this ossicle produces consecutive inflammation of the labyrinth; (3) whether animals hear less with an ankylosed stapes than without one; (4) whether with only the stapes they hear better than without the stapes.

Pigeons were used, as by Kessel in 1876, as the animals upon which experiments were made, since their only ossicle is a stapes, the columella.

The conclusions are as follows :

(1) Erulsion of the stapes in the animals experimented on (rabbite, pigeons, and chickens), is an entirely innocuous operation.

(2) Whether or not the membranes of the oval ${ }^{1}$ and round windows are torn, or whether or not the labyrinth fluid escapes, a new and thicker membrane always forms after the extraction of the stapes or columella.

(3) All animals after the evulsion of their stapes hear well, but not so far, and probably by means of the membranes in the oval and round windows.

(4) Those animals from which the stapes and all other conductors of sound have been removed, but in which the membranes of the oval and round windows and the labyrinth remain normal, hear better than animals in the same condition, but which have ankylosis of the oval window.

(5) In animals which have neither membrana tympani nor columella, lhearing is a little better than in those from which the membrana has been taken, but in which the stapes $h$ as been left.

(6) If partial inflammation occurs about the oval window, the foot-plate of the columella ossifies, and becomes adherent to the edges of the oval window.

1 In birds the columella sits upon a membrane fitted into the oval window. 
(7) If it were possible to perform this operation in man as easily and as well as in birds, it would probably be as inoffensive, where the operation had been thoroughly arranged and executed with rigorous antiseptic precautions.

(8) Almost absolute proof of the preceding conclusion is offered in the case narrated at the beginning of this article.

(9) The auditory ossicles are not indispensable to hearing, as they serve only to reinforce sonorous excitations, which amounts to augmenting the distance of perception.

(10) As a final conclusion, however, it is not proposed to practise removal of the stapes in man; because, first, our experience should be greater, and also because this operation is almost impossible with our present means, as the stapes is nearly always hidden from our view, or, at best, only a portion of it is seen.

In a foot-note the author says: "Avulsion of the stapes has its analogue in extraction of cataract, which oculists have performed daily for one hundred years, because in the latter the object sought for is the removal of the obstacle to the passage of exterior vibrations to the sensorial nerve. To-day, the operation for cataract has not a mortality of even one-half of 1 per cent. The escape of the peri-lymph is still more difficult than that of the humor of the eye, because the former is contained in capillary cavities from which it cannot escape, while the liquid of the eye is less protected and the organ more inclined, perhaps, to subsequent inflammation. It appears, therefore, that when avulsion of the stapes can be rendered practicable, the operation will be for otology - the operation of the future."

\section{OPHTHALMOLOGY.}

UNDER THE CHARGE OF

GEORGE A. BERRY, M.B., F.R.C.S. ED., OPHTHAIMIO SURGEON, EDINBURGH ROYAL INFIRMARY;

AND

EDWARD JACKSON, M.D.,

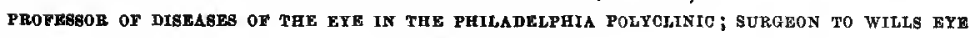
HOSPITA E, ETC.

\section{Optic Neuritis of Gonorrhotil Origin.}

Dr. Panas (La Semaine Médicale, 1890) discusses a case that he regards as being of this description. The patient was a man, aged twenty-nine years, of good muscular development, but anæmic, who had contracted gonorrhoea four years previously, the severe symptoms continuing six months, and including a general rheumatism. The preceding year he had again contracted the 